\title{
Stochastic Modelling of an Aircraft Considering the Possibility of Precautionary Landing Due to Fuel - Filter Clogging
}

\author{
Gurvinder Singh, Gulshan Taneja
}

\begin{abstract}
The present work deals with the stochastic and cost benefit analysis of a helicopter taking into account the situation of precautionary landing caused by blockage in its fuel - filter. The system has been analysed by developing a model and finding the various indices of system effectiveness like mean time to filter clogging, expected up (flying) time, expected number of precautionary landings etc. The regenerative point technique has been used for the purpose. The obtained measures have been further used to analyse the profit generated by the system. Graphical study of the proposed model has also been done. The suggested methodology finds its application in commercial aviation sector.
\end{abstract}

Keywords: Helicopter, Precautionary Landing, Fuel - Filter, Clogging, Regenerative Point Technique

\section{INTRODUCTION}

$\mathrm{O}_{\text {ver the past few decades, the researchers in the field of }}$ reliability modelling have done a lot of research work in a variety of areas and the aviation industry is not an exception in this regard. Many researchers, including [1] - [11], have contributed to the field of aviation research by considering different aircrafts and the systems/devices necessary for adequate functioning of aircrafts. Bigel and Winsten [1] presented an historical overview of the reliability and maintainability growth of F-14A fighter aircraft. Gai et al. [2] analysed the reliability of a dual-redundant engine controller. In [3] and [5], authors modelled and analysed a two engine aeroplane and a GIV Gulf Stream aircraft respectively, using regenerative point technique. Jenab and Rashidi [4] assessed the operational reliability of an aircraft environment control system. Further, the aircraft fuel system and the situation of fuel contamination has also been studied by the researchers [6] - [8]. Besides the above, research work [9] - [12] has been carried out on helicopters considering various aspects and using different methodologies. However, the situation of precautionary landing of a helicopter due to fuel - filter

Revised Manuscript Received on February 05, 2020.

* Correspondence Author

Gurvinder Singh, Assistant Professor of Mathematics, S. J. K. College, Kalanaur, Rohtak, Haryana, India. Email: gsquare29@gmail.com

Gulshan Taneja*, Professor, Department of Mathematics, M. D. University, Rohtak, Haryana, India. Email: drgtaneja@gmail.com

(C) The Authors. Published by Blue Eyes Intelligence Engineering and Sciences Publication (BEIESP). This is an open access article under the CC BY-NC-ND license (http://creativecommons.org/licenses/by-nc-nd/4.0/) clogging, which has not been analysed in earlier studies, also needs to be considered.

A single engine helicopter, with focus on its fuel- filter and bypass valve, has been taken into account in the present study. Engine is the most vital organ of any aircraft which provides the necessary power / thrust to execute a flight. For the engine to keep generating power during a flight, adequate fuel supply to it is quite essential. The fuel from the tank is passed through fuel - filter before entering the engine, in order to ensure that the fuel supplied to the engine is free from contaminants / foreign particles. Another component that forms an integral part of the fuel supply system is the bypass valve which allows the unfiltered fuel to bypass the filter, whenever filter gets clogged. Designing of the bypass valve is such that it opens as and when the pressure of accumulated oil (due to blockage in the filter) reaches a certain limit, thereby preventing fuel starvation. Impending bypass is indicated to the pilot, however the flight is continued as per schedule and helicopter is put under applicable maintenance after landing. In case, the bypass valve gets open during the flight, there is an indication of the same and in order to avoid further damage, precautionary landing is done as soon as practical. After such landing the helicopter is put under the corrective action (as per maintenance manual) for which there is provision of regular maintenance facility.

\section{MATERIALS AND METHODS}

Different performability measures of the studied system have been obtained using regenerative point technique, Laplace transform / convolution and Laplace - Stieltjes transform / convolution. Profit analysis of the system has also been done using the obtained measures. In addition, for calculation work and graphical analysis, software like MATLAB, Mathematica and Excel have been used.

\section{ASSUMPTIONS}

- Initially the helicopter is during flight.

- The situations of impending bypass as well as clogged filter are precisely indicated and bypass valve opens whenever the filter gets clogged.

- Repair / Maintenance work is not feasible during flight and is done only after landing. 
- Precautionary landings are caused by blockage of fuel -filter only (other faults /causes have not been considered in the proposed model). Such landings are followed by inspection (enquiry), under the supervision of concerned authority, to find out the exact cause of problem and actions to be taken accordingly.

- Inspection time includes the time taken by concerned personnel to reach the place of landing.

- Idle time includes the time to carry the helicopter (after maintenance), from place of precautionary landing to the hangar.

- Distribution of flying time till the fuel - filter gets blocked / bypassed, is exponential distribution and all the other distributions are general distributions.

\section{NOTATIONS AND SYMBOLS}

$K_{1}$
$i(t) / I(t)$
$g_{1}(t) / G_{1}(t)$
$g_{2}(t) / G_{2}(t)$
$h_{1}(t) / H_{1}(t)$
$h_{2}(t) / H_{2}(t)$
a(t) /A(t)
b(t) / B(t)

$\mathbf{a}(\mathbf{t}) / \mathbf{A}(\mathbf{t})$
$\mathbf{b}(\mathbf{t}) / \mathbf{B}(\mathbf{t})$

FC $_{\mathbf{i}}(\mathbf{t})$

$F T_{i}(t) / I_{i}(t)$

$\mathbf{M I}_{\mathbf{i}}(\mathbf{t})$

$\mathrm{MC}_{\mathrm{i}}(\mathrm{t})$ rate at which impending bypass is indicated.

rate at which filter gets bypassed (clogged) after the indication of impending bypass

p.d.f. / c.d.f. of inspection (enquiry) time

p.d.f. / c.d.f. of time for which helicopter remains under maintenance following the indication of impending bypass.

p.d.f. / c.d.f. of time for which helicopter remains under maintenance after filter gets clogged (bypassed). p.d.f. /c.d.f. of time required to complete the scheduled flight, after the indication of impending bypass

p.d.f. /c.d.f. of that lapses before precautionary landing is done after fuel - filter gets bypassed

p.d.f. /c.d.f. of scheduled flight duration p.d.f. /c.d.f. of the time for which the operable helicopter remains Idle (because of no demand, unfavorable flying conditions or being involved in scheduled maintenance /overhaul) (or turnaround) maintenance

c.d.f. of the first passage time from regenerative 'i' to the state of filter clogging

probability that the helicopter is in upstate (on flight) / idle state at instant t, given that the system entered regenerative state ' $\mathrm{i}$ ' at $\mathrm{t}=0$

probability that, at instant $t$, the system is under applicable maintenance following the indication of impending bypass, given that the system entered regenerative state ' $\mathrm{i}$ ' at $\mathrm{t}=0$

probability that, at instant $t$, the system is under applicable maintenance following fuel - filter clogging, given that system entered regenerative state p.d.f. /c.d.f. of time taken for preflight
$P L_{i}(t)$

$\mathbf{P}_{0}$

$\mathbf{R}_{\mathbf{f}}$

$\mathbf{C}_{\mathrm{f}}$

$\mathrm{C}_{\mathrm{ib}} / \mathrm{C}_{\mathrm{cl}}$

$\mathbf{C o}_{0}$

$\mathbf{L}$

$* / * *$

(C) / ®

$F_{n} / F_{i b} / F_{c}$

$F_{\text {ibm }}$

$\mathbf{F}_{\mathrm{ci}} / \mathbf{F}_{\mathbf{c m}}$

$F_{\text {id }} / \mathbf{F}_{\mathbf{p f}}$

$\mathbf{V}_{\mathrm{s}} / \mathbf{V}_{\mathrm{o}}$

$\mathbf{V}_{\mathbf{i}} / \mathbf{V}_{\mathbf{m}}$

$\mathbf{V}_{\text {id }} / \mathbf{V}_{\mathbf{p f}}$

$\mathbf{H}_{\mathrm{f}} / \mathbf{H}_{\mathrm{Id}} / \mathbf{H}_{\mathrm{pf}}$

$\mathbf{H}_{\mathbf{i}} / \mathbf{H}_{\mathbf{m}}$ 'i' at $\mathrm{t}=0$

the expected number of precautionary landings in the time interval $(0, t]$, given that the system entered regenerative state ' $\mathrm{i}$ ' at $\mathrm{t}=0$

probability that system sojourns in state 'i,' up to time t.

profit per hour yielded by the system revenue per flight hour

direct operating cost per flight hour (expenses on fuel, lubricant, scheduled maintenance /overhaul etc.)

cost per hour of applicable maintenance following the indication of impending bypass / fuel - filter clogging

indirect operating cost per hour (expenses on staff salaries, insurance premium, regular maintenance facility, office/ hanger rent, and taxes etc.)

goodwill loss and expenses (other than expenses on maintenance) associated per precautionary landing

symbol for Laplace/ Laplace - Stieltjes transform

symbol for Laplace Convolution/ Laplace - Stieltjes Convolution

fuel - filter operating normally/ about to be bypassed /after getting clogged, during flight

fuel-filter under applicable maintenance following the indication of impending bypass

fuel - filter under inspection /applicable maintenance after getting bypassed

fuel - filter during idle / pre-flight state of helicopter

bypass valve in standby / operative (open) mode

bypass valve during inspection/ maintenance of system

bypass valve during idle / pre-flight state of helicopter

helicopter during flight/ in idle state / in preflight state

helicopter under inspection / applicable maintenance

\section{STATE TRANSITION DIAGRAM}

Figure 1 is the state transition diagram depicting the different state transitions. The entry points into the states ' $\mathrm{i}$ ' ( $\mathrm{i}$ $=0$ to 7 ) are regenerative, thus all the states are regenerative. The states $S_{0}, S_{1}$ and $S_{4}$ represent the helicopter during flight. 

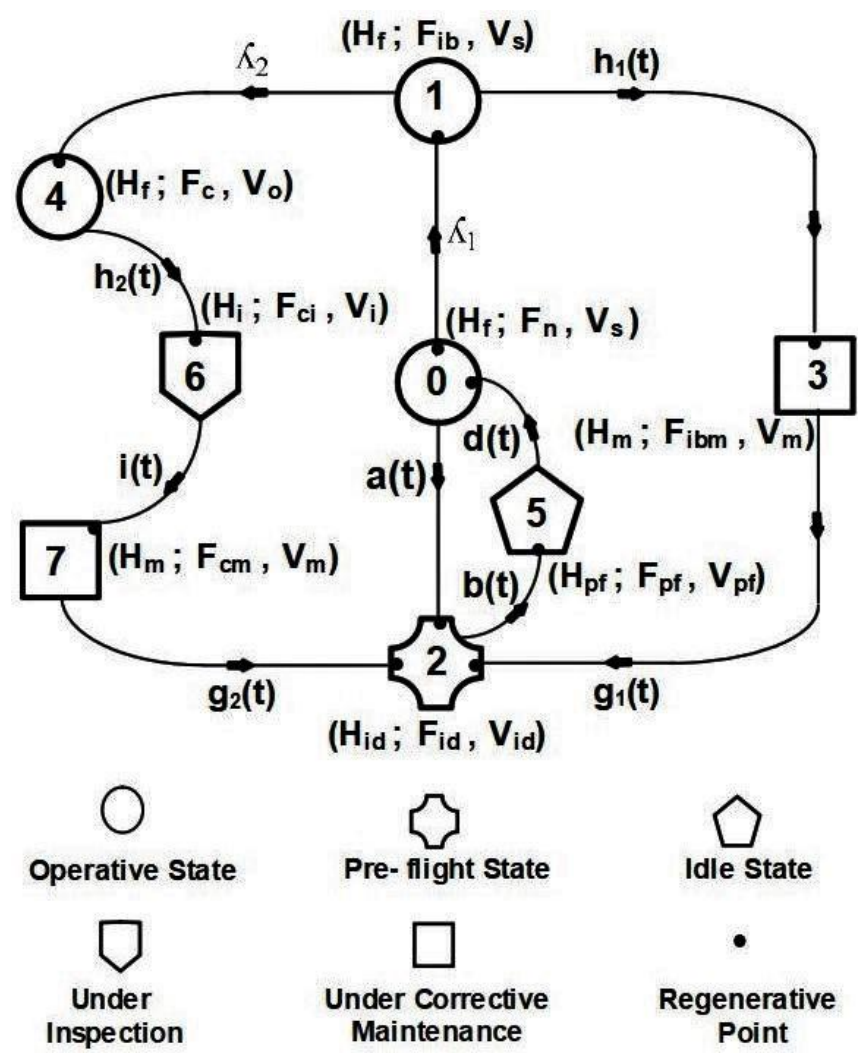

Fig. 1. State transition diagram

\section{TRANSITION TIMES AND PROBABILITIES}

Applying the mathematical arguments, p.d.f. of transition times i.e. $\mathrm{q}_{\mathrm{ijj}}(\mathrm{t})$ are given by:

$$
\begin{aligned}
& q_{01}(\mathrm{t})=\Lambda_{1} \mathrm{e}^{-\Lambda_{1} \mathrm{t}} \overline{\mathrm{A}(\mathrm{t})} \text {, } \\
& q_{02}(t)=\mathrm{e}^{-h_{1} \mathrm{t}} \mathrm{a}(\mathrm{t})_{x} \\
& \mathrm{q}_{13}(\mathrm{t})=\mathrm{e}^{-\mathrm{h}_{2} \mathrm{t}} \mathrm{h}_{1}(\mathrm{t}) \text {. } \\
& \mathrm{q}_{14}(\mathrm{t})=\Lambda_{2} \mathrm{e}^{-h_{2} \mathrm{t}} \overline{\mathrm{H}_{1}(\mathrm{t})} \text {. } \\
& \mathrm{q}_{25}(\mathrm{t})=\mathrm{b}(\mathrm{t}) \text {. } \\
& q_{22}(t)=g_{1}(t){ }_{x} \\
& q_{46}(t)=h_{2}(t) \text {. } \\
& \mathrm{q}_{50}(\mathrm{t})=\mathrm{d}(\mathrm{t}) \text {. } \\
& q_{57}(t)=\mathrm{i}(\mathrm{t}) . \\
& q_{72}(t)=g_{2}(t) \text {. }
\end{aligned}
$$

Using, $\mathrm{p}_{\mathrm{ij}}=\mathrm{q}_{\mathrm{ij}}^{*}(0)$, transition probabilities $\left(\mathrm{p}_{\mathrm{ij}}\right)$ are given by:

$$
\begin{aligned}
& p_{01}=1-a^{*}\left(\Lambda_{1}\right), \\
& p_{02}=a^{*}\left(\Lambda_{1}\right), \\
& p_{13}=h_{1}^{*}\left(\Lambda_{2}\right), \\
& p_{14}=1-h_{1}^{*}\left(\Lambda_{2}\right)_{x} \\
& p_{25}=p_{32}=p_{46}=p_{50}=p_{67}=p_{72}=1 .
\end{aligned}
$$

It follows from the equations (11) to (14) that:

$$
\mathrm{p}_{01}+\mathrm{p}_{02}=\mathrm{p}_{13}+\mathrm{p}_{14}=1
$$

\section{SOJOURN TIMES}

As defined in [5], following expressions for mean sojourn times $\left(\mathrm{L}_{\mathrm{i}}\right)$ and contribution to mean sojourn times $\left(\mathrm{m}_{\mathrm{i}}\right)$ have been obtained:

$\mathrm{b}_{0}=\left\{1-\mathrm{a}^{*}\left(\Lambda_{1}\right)\right\} / \Lambda_{1}$,

$\mathrm{l}_{1}=\left\{1-\mathrm{h}_{1}^{*}\left(\Lambda_{2}\right)\right\} / \Lambda_{2}$, $\mathrm{l}_{2}=-\mathrm{b}^{*}(0)$,

$\mathrm{h}_{\mathrm{a}}=-\mathrm{g}_{1}^{*}(0)$,

$\mathrm{h}_{4}=-\mathrm{h}_{2}^{\mathrm{g}^{*}}(0)$.

$\mathrm{h}_{5}=-\mathrm{d}^{*}(0)$,

$\mathrm{h}_{6}=-\mathrm{i}^{*^{6}}(0)$,

$\mathrm{h}_{7}=-\mathrm{g}_{2}^{6}(0)$,

$\mathrm{m}_{01}=\mathrm{a}^{*}\left({S_{1}}_{1}\right)+\left\{1-\mathrm{a}^{*}\left(\mathrm{~S}_{1}\right)\right\} / \Lambda_{1}$,

$\mathrm{m}_{02}=-\mathrm{a}^{*}\left(\mathrm{C}_{1}\right)$,

$\mathrm{m}_{1 \mathrm{a}}=-\mathrm{h}_{\mathrm{t}}^{*}\left(\mathrm{~K}_{2}\right)$,

$\mathrm{m}_{14}=\mathrm{h}_{1}{ }^{*}\left(\mathrm{~K}_{2}\right)+\left\{1-\mathrm{h}_{1}^{*}\left(\mathrm{~K}_{2}\right)\right\} / \Lambda_{2}$,

$\mathrm{m}_{25}=-\mathrm{b}^{\mathrm{s}^{5}}(0)$,

$\mathrm{m}_{82}=-\mathrm{g}_{1}^{*}(0)$,

$\mathrm{m}_{46}=-\mathrm{h}_{7}^{*}(0)$,

$\mathrm{m}_{50}=-\mathrm{d}^{*}(0)$,

$\mathrm{m}_{67}=-\mathrm{i}^{s^{*}}(0)$,

$\mathrm{m}_{72}=-\mathrm{g}_{2}^{*}(0)$.

From the above expression, it follows that:

$\mathrm{m}_{011}+\mathrm{m}_{02}=\mathrm{ll}_{0}$,

$\mathrm{m}_{13}+\mathrm{m}_{14}=\mathrm{ll}_{1}$,

$\mathrm{m}_{25}=\mathrm{ll}_{2 \mathrm{n}}$,

$\mathrm{m}_{\mathrm{g} 2}=\mathrm{lh}_{\mathrm{g}}$,

$\mathrm{m}_{46}=\mathrm{ln}_{4}$,

$\mathrm{m}_{50}=\mathrm{ll}_{5}$ 。

$\mathrm{m}_{67}=\mathrm{l}_{6}$,

$\mathrm{m}_{72}=\mathrm{ll}_{\mathrm{\gamma}}$.

\section{MEASURES OF SYSTEM EFFECTIVENESS}

\section{A. Mean Time to Filter Clogging (MTFC)}

Reckoning the state of filter clogging, i.e. State '4', as the absorbing state, we get following recursive relations for $\mathrm{FC}_{\mathrm{j}}(\mathrm{t})$ :

$$
\begin{aligned}
& \mathrm{FC}_{0}(\mathrm{t})=\mathrm{O}_{01}(\mathrm{t})(\mathrm{B}) \mathrm{FC}_{1}(\mathrm{t})+\mathrm{O}_{02}(\mathrm{t})(\mathrm{B}) \mathrm{FC}_{2}(\mathrm{t}) \\
& \mathrm{FC}_{1}(\mathrm{t})=\mathrm{Q}_{13}(\mathrm{t}) \text { (B) } \mathrm{FC}_{2}(\mathrm{t})+\mathrm{Q}_{14}(\mathrm{t}) \\
& \mathrm{FC}_{2}(\mathrm{t})=\mathrm{O}_{25}(\mathrm{t}) \text { (8) } \mathrm{FC}_{5}(\mathrm{t}) \\
& \mathrm{FC}_{3}(\mathrm{t})=\mathrm{O}_{32}(\mathrm{t}) \text { (5) } \mathrm{FC}_{2}(\mathrm{t}) \\
& \mathrm{FC}_{5}(\mathrm{t})=\mathrm{O}_{50}(\mathrm{t})(\mathrm{t}) \mathrm{FC}_{0}(\mathrm{t})
\end{aligned}
$$

Taking Laplace - Stieltjes transform of equations (43) - (47) and solving for $\mathrm{FC}_{0}^{* *}(\mathrm{~s})$, we have

$$
\mathrm{FC}_{0}^{* *}(\mathrm{~s})=\mathrm{N}_{1}(\mathrm{~s}) / \mathrm{D}_{1}(\mathrm{~s})
$$

where, $\mathbb{N}_{1}(\mathrm{~s})=q_{01}^{*}(\mathrm{~s}) \mathrm{q}_{14}^{*}(\mathrm{~s})$

$$
D_{1}(\mathrm{~s})=1-\left\{\mathrm{q}_{01}^{*}(\mathrm{~s}) \mathrm{q}_{13}^{*}(\mathrm{~s}) \mathrm{q}_{22}^{*}(\mathrm{~s})+\mathrm{q}_{02}^{*}(\mathrm{~s})\right\} \mathrm{q}_{25}^{*}(\mathrm{~s}) \mathrm{q}_{50}^{*}(\mathrm{~s})
$$

The mean time to filter clogging (MTFC), when the system starts from state ' 0 ', is given by

$$
\mathrm{MTFC}=\lim _{\mathbb{S} \rightarrow 0}\left[\left\{1-\mathrm{FC}_{0}^{* 8}(\mathrm{~s})\right\} / \mathrm{s}\right]=\mathrm{N}_{1} / \mathrm{D}_{1}
$$

where,

$\mathbb{N}_{1}=l_{0}+p_{01} l_{1}+\left(p_{01} p_{13}+p_{02}\right)\left(l_{2}+l_{5}\right)+p_{01} p_{13} l_{2}$ $\mathrm{D}_{1}=\mathrm{p}_{01} \mathrm{p}_{14}$

(51) - (53) 


\section{B. Expected Uptime / Flying Time}

Using the arguments of probability theory and Laplace convolution, we get:

$$
\begin{aligned}
& \mathrm{FT}_{0}(\mathrm{t})=\mathrm{S}_{0}(\mathrm{t})+\mathrm{q}_{01}(\mathrm{t}) \oplus \mathrm{FT}_{1}(\mathrm{t})+\mathrm{q}_{02}(\mathrm{t}) \oplus \mathrm{FT}_{2}(\mathrm{t}) \\
& \mathrm{FT}_{1}(\mathrm{t})=\mathrm{S}_{1}(\mathrm{t})+\mathrm{q}_{13}(\mathrm{t}) \oplus \mathrm{FT}_{3}(\mathrm{t})+\mathrm{q}_{14}(\mathrm{t}) \oplus \mathrm{FT}_{4}(\mathrm{t}) \\
& \mathrm{FT}_{2}(\mathrm{t})=\mathrm{q}_{25}(\mathrm{t}) \oplus \mathrm{FT}_{5}(\mathrm{t}) \\
& \mathrm{FT}_{3}(\mathrm{t})=\mathrm{q}_{32}(\mathrm{t}) \oplus \mathrm{FT}_{2}(\mathrm{t}) \\
& \mathrm{FT}_{4}(\mathrm{t})=\mathrm{S}_{4}(\mathrm{t})+\mathrm{q}_{46}(\mathrm{t}) \oplus \mathrm{FT}_{6}(\mathrm{t}) \\
& \mathrm{FT}_{5}(\mathrm{t})=\mathrm{q}_{50}(\mathrm{t}) \oplus \mathrm{FT}_{0}(\mathrm{t}) \\
& \mathrm{FT}_{6}(\mathrm{t})=\mathrm{q}_{57}(\mathrm{t}) \oplus \mathrm{FT}_{7}(\mathrm{t}) \\
& \mathrm{FT}_{7}(\mathrm{t})=\mathrm{q}_{72}(\mathrm{t}) \oplus \mathrm{FT}_{2}(\mathrm{t})
\end{aligned}
$$

where,

$$
\mathrm{S}_{0}(\mathrm{t})=\mathrm{e}^{-\boldsymbol{L}_{1} \mathrm{t}} \overline{\mathrm{A}(\mathrm{t})}, \mathrm{S}_{1}(\mathrm{t})=\mathrm{e}^{-\boldsymbol{L}_{2} \mathrm{t}} \overline{\mathrm{H}_{1}(\mathrm{t})}, \mathrm{S}_{4}(\mathrm{t})=\overline{\mathrm{H}_{2}(\mathrm{t})}
$$

Following equation is obtained by taking Laplace transform of equations (54) - (61) and solving for $\mathrm{FT}_{0}^{*}(\mathrm{~s})$ :

$$
\mathrm{FT}_{0}^{s}(\mathrm{~s})=\mathrm{N}_{2}(\mathrm{~s}) / \mathrm{D}_{2}(\mathrm{~s})
$$

where,

$$
\begin{aligned}
& N_{2}(\mathrm{~s})=S_{0}^{*}(\mathrm{~s})+q_{01}^{*}(\mathrm{~s}) S_{1}^{*}(\mathrm{~s})+q_{01}^{*}(\mathrm{~s}) q_{14}^{*}(\mathrm{~s}) S_{4}^{*}(\mathrm{~s}) \\
& D_{2}(\mathrm{~s})=1-\left\{q_{01}^{*}(\mathrm{~s}) q_{13}^{*}(\mathrm{~s}) q_{32}^{*}(\mathrm{~s})+q_{01}^{*}(\mathrm{~s}) q_{14}^{*}(\mathrm{~s})\right. \\
& \left.q_{16}^{*}(\mathrm{~s}) q_{63}^{*}(\mathrm{~s}) q_{72}^{*}(\mathrm{~s})+q_{02}^{*}(\mathrm{~s})\right\} q_{25}^{*}(\mathrm{~s}) q_{50}^{*}(\mathrm{~s})
\end{aligned}
$$

In steady-state, the fraction of time for which the helicopter is on flight, is given by

$$
\mathrm{FT}_{0}=\lim _{\mathrm{t} \rightarrow \infty} \mathrm{FT}_{0}(\mathrm{t})=\lim _{\mathrm{s} \rightarrow 0} \mathrm{FT}_{0}^{*}(\mathrm{~s})=\mathrm{N}_{2} / \mathrm{D}_{2}
$$

where,

$$
\begin{aligned}
N_{2}=l_{0} & +p_{01} l_{1}+p_{011} p_{14} l_{4} \\
D_{2}=l_{0} & +p_{01} l_{1}+l_{2}+p_{01} p_{13} l_{19}+l_{5} \\
& +p_{011} p_{14}\left(l_{4}+l_{6}+l_{7}\right)
\end{aligned}
$$

C. Expected Time for Which the Operable Helicopter remains Idle (non-operational)

Proceeding as above, we have:

$$
\begin{aligned}
& \mathrm{ID}_{0}(\mathrm{t})=\mathrm{q}_{01}(\mathrm{t}) \text { (ㄷ) } \mathrm{ID}_{1}(\mathrm{t})+\mathrm{q}_{02}(\mathrm{t}) \text { () } \mathrm{ID}_{2}(\mathrm{t}) \\
& \mathrm{ID}_{1}(\mathrm{t})=\mathrm{q}_{13}(\mathrm{t}) \subseteq \mathrm{ID}_{3}(\mathrm{t})+\mathrm{q}_{14}(\mathrm{t}) \text { (ㄷ) } \mathrm{ID}_{4}(\mathrm{t}) \\
& \mathrm{ID}_{2}(\mathrm{t})=\mathrm{S}_{2}(\mathrm{t})+\mathrm{q}_{25}(\mathrm{t}) \oplus \mathrm{ID}_{5}(\mathrm{t}) \\
& \mathrm{ID}_{3}(\mathrm{t})=\mathrm{q}_{\mathrm{a2}}(\mathrm{t}) \text { (S) } \mathrm{ID}_{2}(\mathrm{t}) \\
& \mathrm{ID}_{4}(\mathrm{t})=\mathrm{q}_{46}(\mathrm{t}) \oplus \mathrm{ID}_{6}(\mathrm{t}) \\
& \mathrm{ID}_{5}(\mathrm{t})=\mathrm{q}_{50}(\mathrm{t}) \oplus \mathrm{ID}_{0}(\mathrm{t}) \\
& \mathrm{ID}_{6}(\mathrm{t})=\mathrm{q}_{57}(\mathrm{t}) \text { (9) } \mathrm{ID}_{7}(\mathrm{t}) \\
& \mathrm{ID}_{7}(\mathrm{t})=\mathrm{q}_{72}(\mathrm{t}) \text { (S) } \mathrm{ID}_{2}(\mathrm{t}) \\
& \text { where, } \mathrm{S}_{2}(\mathrm{t})=\overline{\mathrm{B}(\mathrm{t})}
\end{aligned}
$$

It follows from the above equations that

Where,

$$
\mathrm{ID}_{0}{ }^{*}(\mathrm{~s})=\mathrm{N}_{\mathrm{a}}(\mathrm{s}) / \mathrm{D}_{2}(\mathrm{~s})
$$

$$
\begin{aligned}
N_{g}(s)= & \left\{q_{01}^{*}(s) q_{13}^{*}(s) q_{22}^{*}(s)+q_{01}^{*}(s) q_{14}^{*}(s)\right. \\
& \left.q_{46}^{*}(s) q_{B j}^{*}(s) q_{22}^{*}(s)+q_{02}^{*}(s)\right\} S_{2}^{*}(s)
\end{aligned}
$$

Thus, the fraction of time for which the helicopter remains idle, is given by

$$
\mathrm{ID}_{0}=\lim _{\mathrm{t} \rightarrow \mathrm{s}} \mathrm{ID}_{0}(\mathrm{t})=\lim _{\mathrm{g} \rightarrow 0} \mathrm{~s} \mathrm{ID}_{0}^{*}(\mathrm{~s})=\mathbb{N}_{\mathrm{g}} / \mathrm{D}_{2}
$$

where, $\mathbb{N}_{3}=\mathrm{ll}_{2}$
(82) - (83)

D. Expected Time for which the System is under Applicable Maintenance Following the Indication of Impending Bypass

To find the required time, we have obtained the following recursive relations for $\mathrm{MI}_{\mathrm{i}}(\mathrm{t})$ :

$$
\begin{aligned}
& \mathrm{MI}_{0}(\mathrm{t})=\mathrm{q}_{01}(\mathrm{t}) \oplus \mathrm{MI}_{1}(\mathrm{t})+\mathrm{q}_{02}(\mathrm{t}) \oplus \mathrm{MI}_{2}(\mathrm{t}) \\
& \mathrm{MI}_{1}(\mathrm{t})=\mathrm{q}_{13}(\mathrm{t}) \oplus \mathrm{MI}_{3}(\mathrm{t})+\mathrm{q}_{14}(\mathrm{t}) \oplus \mathrm{MI}_{4}(\mathrm{t}) \\
& \mathrm{MI}_{2}(\mathrm{t})=\mathrm{q}_{25}(\mathrm{t}) \text { (c) } \mathrm{MI}_{5}(\mathrm{t}) \\
& \mathrm{MI}_{3}(\mathrm{t})=\mathrm{S}_{\mathrm{g}}(\mathrm{t})+\mathrm{q}_{32}(\mathrm{t}) \text { (C) } \mathrm{MI}_{2}(\mathrm{t}) \\
& \mathrm{MI}_{4}(\mathrm{t})=\mathrm{q}_{46}(\mathrm{t}) \text { (c) } \mathrm{MI}_{6}(\mathrm{t}) \\
& \mathrm{MI}_{5}(\mathrm{t})=\mathrm{q}_{50}(\mathrm{t}) \text { () } \mathrm{MI}_{0}(\mathrm{t}) \\
& \mathrm{MI}_{6}(\mathrm{t})=\mathrm{q}_{57}(\mathrm{t}) \text { () } \mathrm{MI}_{7}(\mathrm{t}) \\
& \mathrm{MI}_{7}(\mathrm{t})=\mathrm{q}_{72}(\mathrm{t}) \text { (c) } \mathrm{MI}_{2}(\mathrm{t}) \\
& \text { Where, } \mathrm{S}_{3}(\mathrm{t})=\overline{\mathrm{G}_{1}(\mathrm{t})}
\end{aligned}
$$

Proceeding in the similar manner, as before, we have

$$
\mathrm{MI}_{0}{ }^{*}(\mathrm{~s})=\mathrm{N}_{4}(\mathrm{~s}) / \mathrm{D}_{2}(\mathrm{~s})
$$

where,

$$
\mathbb{N}_{4}(s)=q_{01}^{*}(s) q_{13}^{*}(s) S_{3}^{*}(s)
$$

Therefore, we get

$$
\mathrm{MI}_{0}=\lim _{\mathrm{t} \rightarrow \infty} \mathrm{MI}_{0}(\mathrm{t})=\lim _{\mathrm{g} \rightarrow 0} \mathrm{~s} \mathrm{MI}_{0}^{*}(\mathrm{~s})=\mathrm{N}_{4} / \mathrm{D}_{2}
$$

where, $\mathrm{N}_{4}=\mathrm{p}_{01} \mathrm{p}_{13} \mathrm{l}_{3}$

E. Expected Time for which the System is under Applicable Maintenance after the Fuel - filter gets Bypassed (Clogged)

Again proceeding in the similar fashion, we get:

$$
\begin{aligned}
& \mathrm{MC}_{0}(\mathrm{t})=\mathrm{q}_{01}(\mathrm{t}) \oplus \mathrm{MC}_{1}(\mathrm{t})+\mathrm{q}_{02}(\mathrm{t}) \oplus \mathrm{MC}_{2}(\mathrm{t}) \\
& M_{1}(t)=q_{13}(t) \oplus M_{3}(t)+q_{14}(t) \text { (s) } M_{4}(t) \\
& \mathrm{MC}_{2}(\mathrm{t})=\mathrm{q}_{25}(\mathrm{t}) \oplus \mathrm{MC}_{5}(\mathrm{t}) \\
& \mathrm{MC}_{3}(\mathrm{t})=\mathrm{q}_{22}(\mathrm{t}) \text { (S) } \mathrm{MC}_{2}(\mathrm{t}) \\
& \mathrm{MC}_{4}(\mathrm{t})=\mathrm{q}_{46}(\mathrm{t}) \text { (s) } \mathrm{MC}_{6}(\mathrm{t}) \\
& \mathrm{MC}_{5}(\mathrm{t})=\mathrm{q}_{50}(\mathrm{t}) \text { (S) } \mathrm{MC}_{0}(\mathrm{t}) \\
& \mathrm{MC}_{6}(\mathrm{t})=\mathrm{q}_{57}(\mathrm{t}) \text { (5) } \mathrm{MC}_{7}(\mathrm{t}) \\
& \mathrm{MC}_{7}(\mathrm{t})=\mathrm{S}_{7}(\mathrm{t})+\mathrm{q}_{72}(\mathrm{t}) \text { (9) } \mathrm{MC}_{2}(\mathrm{t}) \\
& \text { Where, } \mathrm{S}_{7}(\mathrm{t})=\overline{\mathrm{G}_{2}(\mathrm{t})}
\end{aligned}
$$

Following expression for $\mathrm{MC}_{0}{ }^{*}(\mathrm{~s})$ has been obtained from the equations (97) - (104):

$$
\begin{gathered}
\mathrm{MC}_{0}^{*}(\mathrm{~s})=\mathrm{N}_{5}(\mathrm{~s}) / \mathrm{D}_{2}(\mathrm{~s}) \\
\text { where, } \mathrm{N}_{5}(\mathrm{~s})=\mathrm{q}_{01}^{*}(\mathrm{~s}) \mathrm{q}_{14}^{*}(\mathrm{~s}) \mathrm{q}_{46}^{*}(\mathrm{~s}) \mathrm{q}_{57}^{*}(\mathrm{~s}) \mathrm{S}_{7}^{*}(\mathrm{~s})
\end{gathered}
$$

Thus, we have

$$
\mathrm{MC}_{0}=\lim _{\mathrm{t} \rightarrow \mathrm{\infty}} \mathrm{MC}_{0}(\mathrm{t})=\lim _{\mathrm{g} \rightarrow 0} \mathrm{MC}_{0}^{*}(\mathrm{~s})=\mathrm{N}_{5} / \mathrm{D}_{2}
$$

Where, $\mathbb{N}_{5}=p_{01} p_{14} l_{5}$

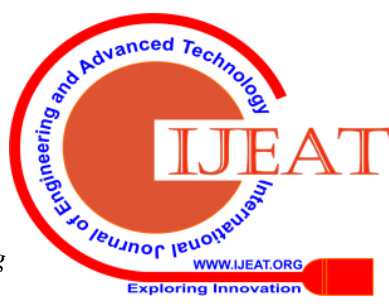




\section{F. Expected Number of Precautionary Landings}

Applying arguments as above, we have the following recursive relations for $\mathrm{PL}_{\mathrm{i}}(\mathrm{t})$ :

$$
\begin{aligned}
& \mathrm{PL}_{0}(\mathrm{t})=\mathrm{Q}_{01}(\mathrm{t}) \text { (5) } \mathrm{PL}_{1}(\mathrm{t})+\mathrm{Q}_{02}(\mathrm{t}) \text { (5) } \mathrm{PL}_{2}(\mathrm{t}) \\
& \mathrm{PL}_{1}(\mathrm{t})=\mathrm{Q}_{13}(\mathrm{t}) \text { (5) } \mathrm{PL}_{2}(\mathrm{t})+\mathrm{Q}_{14}(\mathrm{t}) \text { (5) } \mathrm{PL}_{4}(\mathrm{t}) \\
& \mathrm{PL}_{2}(\mathrm{t})=\mathrm{Q}_{25}(\mathrm{t}) \text { (5) } \mathrm{PL}_{5}(\mathrm{t}) \\
& \mathrm{PL}_{2}(\mathrm{t})=\mathrm{Q}_{36}(\mathrm{t}) \text { (5) } \mathrm{PL}_{6}(\mathrm{t}) \\
& \mathrm{PL}_{4}(\mathrm{t})=\mathrm{Q}_{46}(\mathrm{t}) \text { (5) }\left\{1+\mathrm{PL}_{5}(\mathrm{t})\right\} \\
& \mathrm{PL}_{5}(\mathrm{t})=\mathrm{Q}_{50}(\mathrm{t}) \text { (5) } \mathrm{PL}_{0}(\mathrm{t}) \\
& \mathrm{PL}_{6}(\mathrm{t})=\mathrm{Q}_{67}(\mathrm{t}) \text { (5) } \mathrm{PL}_{7}(\mathrm{t}) \\
& \mathrm{PL}_{7}(\mathrm{t})=\mathrm{Q}_{72}(\mathrm{t}) \text { (5) } \mathrm{PL}_{2}(\mathrm{t})
\end{aligned}
$$

Advancing as earlier, we get

$$
\mathrm{PL}_{0}{ }^{* *}(\mathrm{~s})=\mathrm{N}_{6}(\mathrm{~s}) / \mathrm{D}_{2}(\mathrm{~s})
$$

where,

$$
N_{6}(s)=q_{01}^{*}(s) q_{14}^{*}(s) q_{46}^{*}(s)
$$

Thus, expected number of precautionary landings per unit time is given by

$$
\mathrm{PL}_{0}=\lim _{\mathrm{t} \rightarrow \infty}\left[\mathrm{PL}_{0}(\mathrm{t}) / \mathrm{t}\right]=\lim _{\mathbb{s} \rightarrow 0} \mathrm{PL}_{0}^{* \bullet}(\mathrm{s})=\mathbb{N}_{6} / \mathrm{D}_{2}
$$$$
\text { where, } \mathbb{N}_{6}=p_{01} p_{14}
$$

\section{PROFIT ANALYSIS}

Profit yielded by the system can be expressed as: $\mathrm{P}_{0}=\left(\mathrm{R}_{\mathrm{f}}-\mathrm{C}_{\mathrm{f}}\right) \mathrm{FT}_{0}-\left\{\mathrm{C}_{\mathrm{ib}} \mathrm{MI}_{0}+\mathrm{C}_{\mathrm{cl}} \mathrm{MC}_{0}+\mathrm{L} \mathrm{PL}_{0}+\mathrm{C}_{\mathrm{o}}\right\}$

where, $\mathrm{R}_{\mathrm{f}}, \mathrm{C}_{\mathrm{f}}, \mathrm{C}_{\mathrm{ib}}, \mathrm{C}_{\mathrm{cl}}, \mathrm{C}_{\mathrm{o}}, \mathrm{L}$ are as defined in Section IV and expressions for $\mathrm{FT}_{0}, \mathrm{MI}_{0}, \mathrm{MC}_{0}, \mathrm{PL}_{0}$ have been obtained in previous section.

Every commercial operator desires to earn certain minimum profit. If $\mathrm{P}_{\min }$ denotes the desired minimum profit, then it follows from equation (122) that:

- $\mathrm{P}_{0} \geq \mathrm{P}_{\min }$ if and only if $\mathrm{R}_{\mathrm{f}} \geq \mathrm{X}_{1} / \mathrm{FT}_{0}$

where,

$\mathrm{X}_{\mathrm{I}}=\mathrm{P}_{\min }+\left\{\mathrm{C}_{\mathrm{f}} \mathrm{FT}_{0}+\mathrm{C}_{\mathrm{ib}} \mathrm{MI}_{0}+\mathrm{C}_{\mathrm{cl}} \mathrm{MC}_{0}+\mathrm{L} \mathrm{PL}_{0}+\mathrm{C}_{0}\right\}$

- $\mathrm{P}_{0} \geq \mathrm{P}_{\min }$ if and only if $\mathrm{C}_{0} \leq \mathrm{X}_{2}$

\begin{tabular}{|c|c|c|c|c|c|}
\hline \multicolumn{4}{|c|}{$\begin{array}{c}\text { Rates } \\
\text { (per hour) }\end{array}$} & \multicolumn{2}{|c|}{$\begin{array}{c}\text { Revenue/ Costs / Loss } \\
\text { (INR) }\end{array}$} \\
\hline$\alpha$ & 1 & $\kappa_{1}$ & $0 . .00067$ & $\mathrm{R}_{\mathrm{f}}$ & 90000 \\
\hline$\beta$ & 0.0635 & $\kappa_{2}$ & 0.25 & $\mathrm{P}_{\min }$ & 1000 \\
\hline$\gamma_{1}$ & 1.2 & $\delta$ & 0.0028 & $\overline{C_{f}}$ & 22000 \\
\hline$\gamma_{2}$ & 3 & $\delta_{1}$ & 1 & $\mathrm{C}_{\mathrm{ib}}$ & 7000 \\
\hline \multirow[t]{3}{*}{$\sigma$} & \multirow[t]{3}{*}{1.5} & \multirow[t]{3}{*}{$\delta 2$} & \multirow[t]{3}{*}{0.0083} & $\mathrm{C}_{\mathrm{cl}}$ & 2100 \\
\hline & & & & $\mathrm{C}_{\mathrm{o}}$ & 2870 \\
\hline & & & & $\mathrm{L}$ & 50000 \\
\hline
\end{tabular}

where,

$\mathrm{X}_{2}=\left(\mathrm{R}_{\mathrm{f}}-\mathrm{C}_{\mathrm{f}}\right) \mathrm{FT}_{0}-\left\{\mathrm{C}_{\mathrm{ib}} \mathrm{MI}_{0}+\mathrm{C}_{\mathrm{cl}} \mathrm{MC}_{0}+\mathrm{L} \mathrm{PL}_{0}+\mathrm{P}_{\min }\right\}$

\section{GRAPHICAL ANALYSIS}

Following particular case, wherein all the time distributions are assumed to be exponential, is considered for the purpose of graphical analysis of model under consideration:

$$
\begin{array}{lll}
a(t)=\alpha e^{-\alpha t} & b(t)=\beta e^{-\beta t} & d(t)=\sigma e^{-\sigma t} \\
h_{1}(t)=\gamma_{1} e^{-\gamma_{1} t} & h_{2}(t)=\gamma_{2} e^{-\gamma_{2} t} & i(t)=\delta e^{-\delta t} \\
g_{1}(t)=\delta_{1} e^{-\delta_{1} t} & g_{2}(t)=\delta_{2} e^{-\delta_{2} t} &
\end{array}
$$

Assumed values have been assigned to various parameters as per Table I.
Different graphs demonstrating the behavior of MTFC, expected uptime and profit, have been plotted, as shown below:

a) Varying the values of $K_{1}$ and $K_{2}$ while keeping the other parameters constant as per Table 1 , the following graph of MTFC versus $K_{1}$, for different values of $\kappa_{2}$, has been plotted:

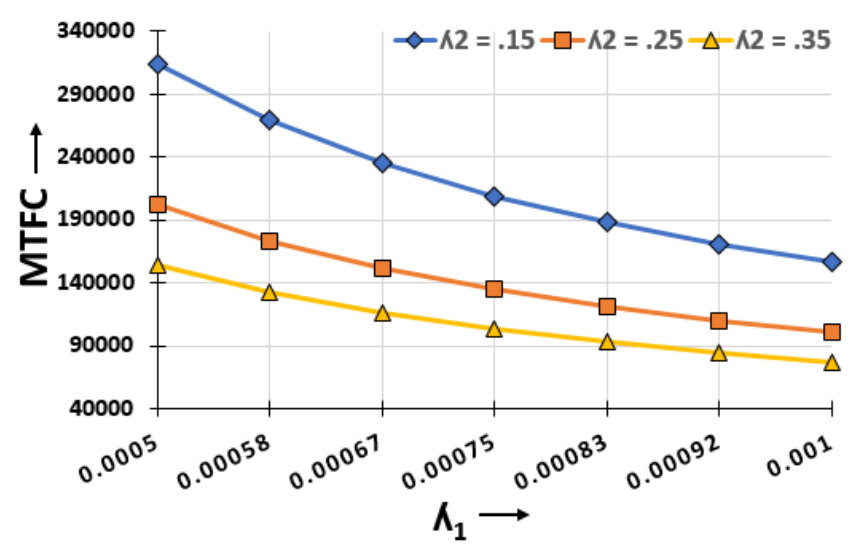

Fig. 2. Graph of MTFC vs. $\kappa_{1}$

b) Fig. 3, given below, shows change in expected uptime (flying time) with variation in value of $\beta$, for different values of $\kappa_{1}$ :

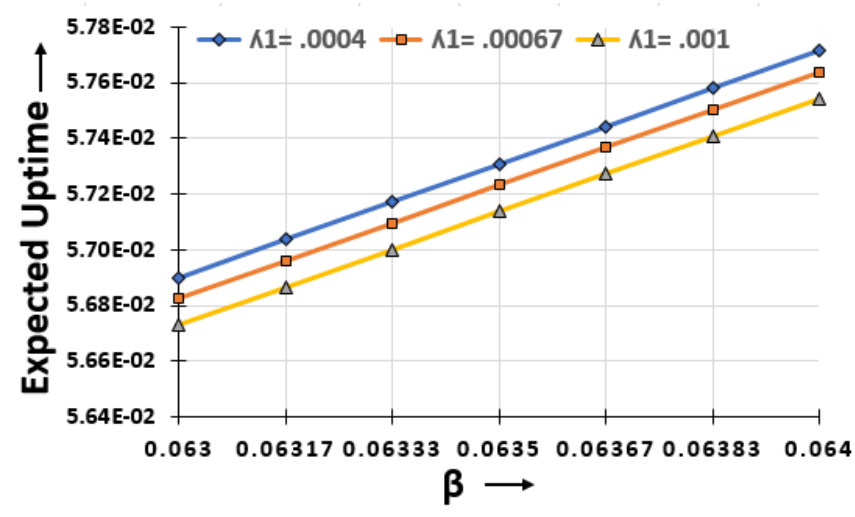

Fig. 3. Graph of FTo vs. $\beta$

c) The following graph depicts the behavior of profit per hour with variation in value of revenue per flight hour, for different values of $\alpha$ :

Table- I: Assumed numerical values 


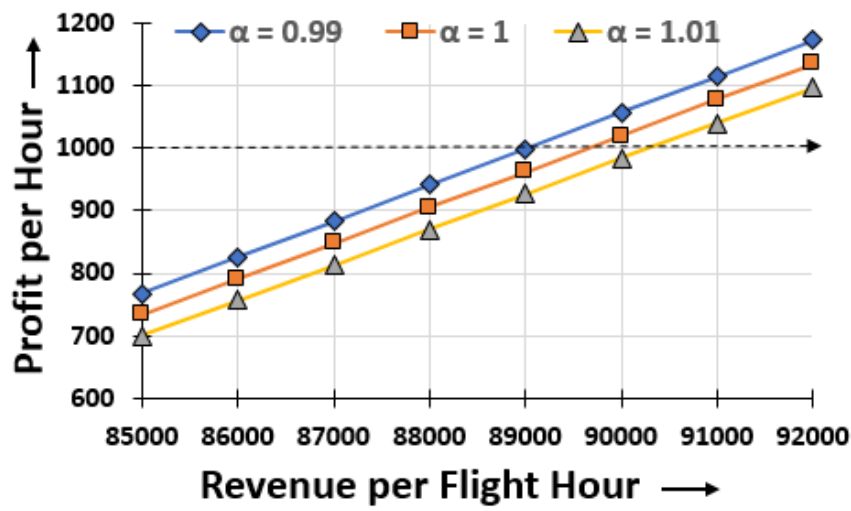

Fig. 4. Graph of $P_{0}$ vs. $R_{f}$

d) Again, varying the values of $C_{o}$ and $\beta$ and taking the numerical values of other parameters as per Table 1 , the following graph has been plotted:

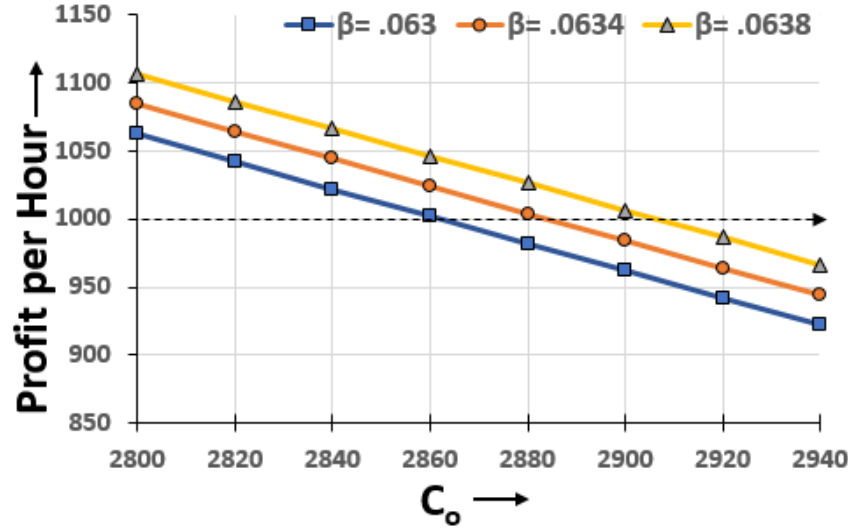

Fig. 5. Graph of $P_{0}$ vs. $C_{o}$

\section{RESULT AND DISCUSSION}

From the graphical analysis as done in the above Section, following may be observed:

1) MTFC, uptime /flying time and profit vary in expected manner with respect to change in different parameters as shown in Figures 2 to 5.

2) Figures 4 and 5 reveal the numerical outcomes as tabulated below:

Table - II: Obtained cut - off values with interpretations

\begin{tabular}{|c|c|c|c|c|}
\hline \multirow[b]{2}{*}{$\begin{array}{l}\text { S. } \\
\text { N. }\end{array}$} & \multirow{2}{*}{$\begin{array}{c}\text { Fixed parameters } \\
\text { (with values as per Table } \\
\text { I) }\end{array}$} & \multirow[b]{2}{*}{$\begin{array}{c}\text { Varied } \\
\text { parameters }\end{array}$} & \multicolumn{2}{|c|}{ Cut - off value } \\
\hline & & & For & $\begin{array}{c}\text { Value } \\
\text { obtained } \\
\text { (Approx.) }\end{array}$ \\
\hline \multirow{3}{*}{1} & \multirow{3}{*}{$\begin{array}{c}\beta, \gamma_{1}, \gamma_{2}, \sigma, \delta, \delta_{1}, \delta_{2}, \Lambda_{1}, \Lambda_{2}, \\
\mathrm{C}_{\mathrm{f}}, \mathrm{C}_{\mathrm{ib}}, \mathrm{C}_{\mathrm{cl}}, \mathrm{C}_{\mathrm{o}}, \mathrm{L}\end{array}$} & \multirow{3}{*}{$\alpha$ and $R_{f}$} & $\begin{array}{c}\alpha= \\
0.99\end{array}$ & $\begin{array}{c}\mathrm{R}_{\mathrm{f}}= \\
89025.1342\end{array}$ \\
\hline & & & $\alpha=1$ & $\begin{array}{c}\mathrm{R}_{\mathrm{f}}= \\
89660.0652\end{array}$ \\
\hline & & & $\begin{array}{l}\alpha= \\
1.01\end{array}$ & $\begin{array}{c}\mathrm{R}_{\mathrm{f}}= \\
90294.9962\end{array}$ \\
\hline \multirow{3}{*}{2} & \multirow{3}{*}{$\begin{array}{c}\alpha, \gamma_{1}, \gamma_{2}, \sigma, \delta, \delta_{1}, \delta_{2}, \Lambda_{1}, \kappa_{2}, \\
\mathrm{R}_{\mathrm{f}}, \mathrm{C}_{\mathrm{f}}, \mathrm{C}_{\mathrm{ib}}, \mathrm{C}_{\mathrm{cl}}, \mathrm{L}\end{array}$} & \multirow{3}{*}{$\beta$ and $C_{o}$} & $\begin{array}{c}\beta= \\
.0630\end{array}$ & $\begin{array}{c}\mathrm{C}_{\mathrm{o}}= \\
2861.8266\end{array}$ \\
\hline & & & $\begin{array}{c}\beta= \\
.0634\end{array}$ & $\begin{array}{c}\mathrm{C}_{0}= \\
2883.9325\end{array}$ \\
\hline & & & $\begin{array}{c}\beta= \\
.0638\end{array}$ & $\begin{array}{c}\mathrm{C}_{\mathrm{o}}= \\
2906.0109\end{array}$ \\
\hline
\end{tabular}

\section{CONCLUSION}

The studied system has been modelled and analysed taking into consideration the situation of precautionary landing due to fuel - filter clogging. Various measures of the system performance and profit equation have been obtained in Sections VIII and IX. For numerical illustration and graphical analysis of the proposed model, particular case has been considered. The effect of variation in different parameters on MTFC, expected uptime and profit has been graphically observed and discussed in Sections $\mathbf{X}$ and XI. In addition to above, for getting the desired minimum profit, the cut-off values for some parameters have also been obtained. The suggested methodology can be adopted by the commercial aircraft operators to accomplish the same or to find the cut -off values related to other parameters of interest.

2. E. Gai, J. V. Harrison, R.H. Luppold, Reliability Analysis of a Dual-Redundant Engine Controller, IEEE Transactions on Reliability, vol. R-32, 1983, 14-20.

3. L.R. Goel, P. Gupta, Analysis of a Two-Engine Aeroplane Model with Two Types of Failure and Preventive Maintenance, Microelectron. Reliab., vol. 24(4), 1984, 663-666.

4. K. Jenab, K. Rashidi, Operational Reliability Assessment of an Aircraft Environmental Control System, Reliability Engineering and System Safety, vol. 94, 2009, 456-462.

5. M. N. Al Alawi, A.G. Mathew, J. Collington, Attaining Zero Failure Performance for GIV Gulfstream Aircraft through Reliability Modeling and Analysis, IJASETR, vol. 1(2), 2012, 1-9.

6. S. Sharma, P. Singh, R. Sinha, K.P. Kaurase, Review of Aircraft Fuel System, IJARIIE, vol. 1(1), 2015, 32-38.

7. C. R. Babu, S. M. Shareef, Flow Test to Analyze Fuel Filter Element of an Aircraft, International Journal for Modern Trends in Science and Technology, vol. 3(10), 2017, 127-132.

8. B. Pour MJ, Radice G, Fuel Contamination on the Large Transport Airplanes, Journal of Aeronautical \& Aerospace Engineering, vol. 6(4), 2017, 1-11.

9. S. Castillo-Rivera, M. Tomas-Rodriguez, Helicopter Modelling and Study of the Accelerated Rotor, Advances in Engineering Software, vol. 115, 2018, 52-65.

\section{REFERENCES}

1. G. Bigel, J. Winsten, Reliability and Maintainability Growth of A Modern, High Performance Aircraft, The F-14A, Microelectronics and Reliability, vol. 19, 1979, 31-38.

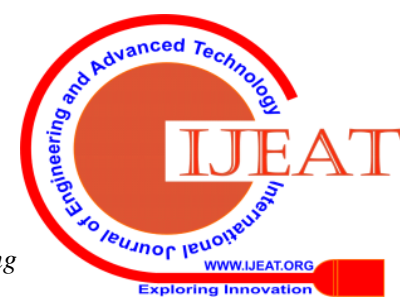


10. A. Tamer, V. Muscarello, P. Masarati, G. Quaranta, Evaluation of Vibration Reduction Devices for Helicopter Ride Quality Improvement, Aerospace Science Technology, vol. 95, 2019, 105456.

11. H. Ji, R. Chen, and P. Li, Real-time Simulation Model for Helicopter Fight Task Analysis in Turbulent Atmospheric Environment, Aerospace Science and Technology, vol. 92, 2019, 289-299.

12. Y. Ren, K. Li, H. Ye, Modeling and Anti-swing Control for a Helicopter Slung-load System, Applied Mathematics and Computation, vol. 372, 2020, 124990.

\section{AUTHORS PROFILE}

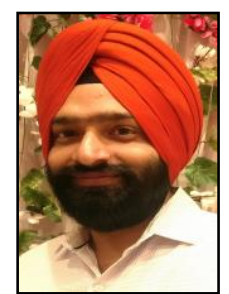

Gurvinder Singh, is working as an Assistant Professor of Mathematics at S. J. K. College, Kalanaur, Rohtak, Haryana, India since last 13 years. He passed M.Sc. and M. Phil. (Mathematics) from the the Department of Mathematics, M. D. University, Rohtak, and is presently pursuing Ph.D. programme from the same university. The area of his research work is Reliability Modelling. He qualified the joint CSIR-UGC National Eligibility Test (NET) for lectureship in the year 2002. He has participated in over 15 Seminars / Conferences / Workshops.

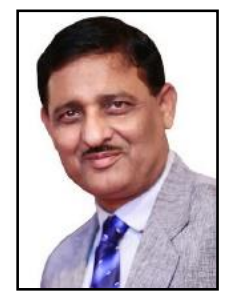

Gulshan Taneja, is a Professor in the Department of Mathematics, M. D. University, Rohtak, Haryana, India with 30 years of teaching and research experience. He is an Associate Editor/Editor/Reviewer of some journals. He has supervised $25 \mathrm{PhD} / \mathrm{MPhil}$ dissertations and completed two research projects successfully. He has got published over 100 research papers in journals of repute and over 35 in the proceedings of conferences. He has contributed in over 50 conferences. He has authored six books and edited six books/proceedings of conferences. He is an office bearer/member of various academic societies/associations. His areas of interest are reliability modelling, queuing theory and information theory. 